03

\title{
Геометрическая оптика твист-ориентированного нематического жидкого кристалла с анизотропным поглощением
}

\author{
(C) А.О. Овакимян, А.Э. Айвазян, Л.С. Асланян \\ Ереванский государственный университет, физический фракультет, \\ 0025 Ереван, Республика Армения \\ e-mail: harutyun.hovakimyan@ysu.am
}

Поступила в редакцию 08.04.2019 г.

В окончательной редакции 08.04.2019 г.

Принята к публикации 11.06.2019 г.

\begin{abstract}
Проанализирована пространственная динамика поляризованного света в плавнонеоднородной анизотропной среде с примесью анизотропно поглощающего красителя. В качестве такой среды рассматривался твист-ориентированный нематический жидкий кристалл с плеохроичным красителем. В геометрооптическом приближении получена система связанных укороченных уравнений относительно декартовых компонент электрической составляющей световой волны. Аналитически решена система связанных комплексных уравнений и проанализировано поведение линейно поляризованной волны в такой среде в случае как поглощения, так и усиления. Обнаружено асимметричное влияние этих двух процессов на пространственную динамику распространения света в среде.
\end{abstract}

Ключевые слова: плеохроизм, переход Фредерикса, тензор диэлетрической проницаемости, вектор Джонса.

DOI: $10.21883 /$ OS.2019.11.48516.129-19

\section{Введение}

Распространение света в анизотропных средах в настоящее время является хорошо исследованным разделом оптики [1-3]. Ситуация усложняется, если среда обладает также и анизотропным поглощением. В однородных кристаллах взаимное расположение главных осей тензора диэлектрической проницаемости и поглощения являются произвольными, и анализ распространения световой волны является сложной задачей. Решение, однако, существенно упрощается в случае совпадения направления главных осей этих двух тензоров [3].

Появление сред с управляемой ориентацией оптической оси намного усложнило задачу [4] но, с другой стороны, существенно расширило круг исследуемых явлений. В отличие от однородных неоднородные анизотропные среды способны управляемо изменять характеристики световых полей, что и приводит к их широкому применению в различных устройствах управления параметрами излучения [5-7]. К числу таких сред относятся жидкие кристаллы (ЖК), в которых ориентацию оптической оси возможно плавно регулировать с помощью внешних воздействий. Если в такой неоднородно анизотропной среде присутствуют анизотропно поглощающие молекулы (плеохроичные красители), то в такой комбинированной плавнонеоднородной анизотропной среде с поглощением аналитическое решение возможно, если только деформации тензора диэлектрической проницаемости и поглощения не нарушают совпадение их главных осей.

В анизотропной плавнонеоднородной среде нормальные волны подчиняются связанным уравнениям. В ре- зультате параметры света меняются вдоль направления распространения, и задача сводится к выявлению характера этих изменений в зависимости от свойств среды. Хотя аналитическое решение системы связанных уравнений в таких средах связано с определенными трудностями даже при возможности применения приближенных методов, анализ пространственной динамики волновых характеристик света в плавнонеоднородной анизотропной среде с поглощением представляет определенный интерес не только в оптике, но и в квантовой механике, в физике плазмы и др. [8-10].

Исследованию распространения света в неоднородно анизотропной среде посвящено большое количество работ $[11,12]$. В более ранних работах нами была показана возможность решения системы связанных уравнений в твист-ориентированном нематическом жидком кристалле (НЖК) путем перехода во вращающуюся систему координат [13-15].

Целью настоящей работы является систематический анализ пространственной динамики света в плавнонеоднородной анизотропной среде с анизотропным поглощением. В качестве такой среды рассматривается твист-ориентированный НЖК с примесью плеохроичных молекул. Отметим, что интерес к такой смеси повысился в связи с обнаружением явления понижения порога перехода Фредерикса и усиления ориентационной оптической нелинейности в НЖК [16,17]. В НЖК явление анизотропии поглощения также интересно тем, что можно осуществлять переключение между двумя состояниями с различными спектрами пропускания, подавая на ЖК внешнее управляющее напряжение, т.е. наблюдая переход Фредерикса. На этом принципе работают 


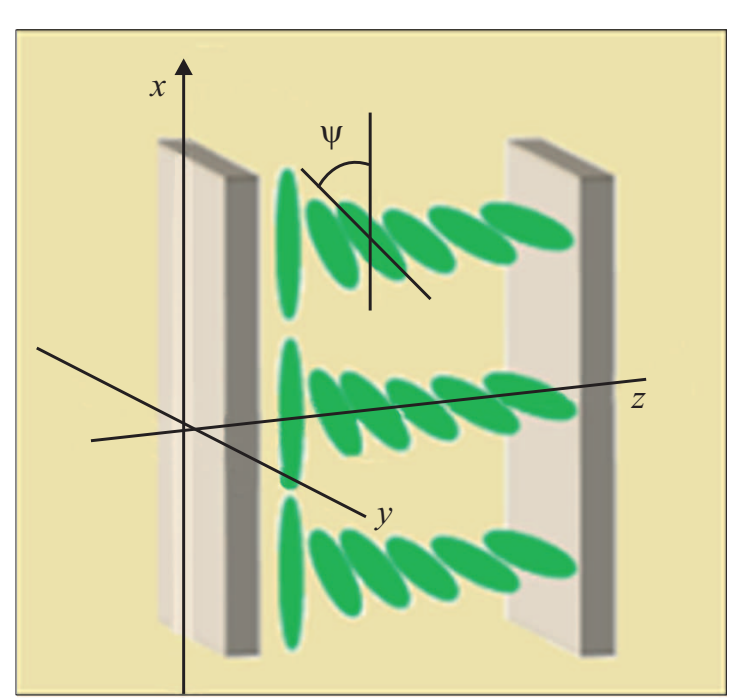

Рис. 1. Геометрия задачи.

многие цветные устройства отображения информации на ЖК.

\section{Описание базисной модели}

Пусть плоская монохроматическая волна распространяется в среде, которая может обладать неоднородной анизотропией. Примем, что оси лабораторной системы координат $x, y$ совпадают с направлениями необыкновенной и обыкновенной волн на входе, а $z$ - координата направлена вдоль распространения волны (рис. 1).

Существует большое число веществ, для которых поглощение света зависит от направления распространения и состояния поляризации. Явление изменения оптического пропускания вещества в зависимости от направления распространения и состояния поляризации излучения, падающего на это вещество, получило название плеохроизма. Плеохроизм обусловлен анизотропией поглощения излучения, падающего на вещество.

Рассмотрим случай, когда в среде, кроме неоднородности анизотропии, может присутствовать также неоднородное анизотропное поглощение. Согласно $[11,18]$, представим тензор диэлектрической проницаемости такой среды в виде суммы двух эрмитово сопряженных тензоров:

$$
\tilde{\varepsilon}_{i j}(z)=\varepsilon_{i j}(z)+i \tau_{i j}(z) .
$$

Тензор $\varepsilon_{i j}(z)$ характеризует диэлектрические свойства среды (НЖК), а $\tau_{i j}(z)$ - поглощение (краситель). В свою очередь, $\varepsilon_{i j}(z)$ и $\tau_{i j}(z)$ бывает удобнее представить в виде суммы симметричных и антисимметричных составляющих:

$$
\varepsilon_{i j}=\varepsilon_{i j, s}+i \varepsilon_{i j, a}, \quad \tau_{i j}=\tau_{i j, s}+i \tau_{i j, a},
$$

где

$$
\varepsilon_{i j, s}=\frac{\varepsilon_{i j}+\varepsilon_{j i}}{2}, \quad \varepsilon_{i j, a}=\frac{\varepsilon_{i j}-\varepsilon_{j i}}{2 i}
$$

$$
\tau_{i j, s}=\frac{\tau_{i j}+\tau_{j i}}{2}, \quad \tau_{i j, a}=\frac{\tau_{i j}-\tau_{j i}}{2 i} .
$$

Симметричность $\varepsilon_{i j, s}$ и $\tau_{i j, s}$, а также антисимметричность $\varepsilon_{i j, a}$ и $\tau_{i j, a}$ вытекают из эрмитовости тензоров $\varepsilon_{i j}(z)$ и $\tau_{i j}(z)$. Тогда двумерный тензор диэлектрической проницаемости рассматриваемой среды представляется в виде

$$
\tilde{\varepsilon}_{i j}=\left(\varepsilon_{i j, s}-\tau_{i j, a}\right)+i\left(\varepsilon_{i j, a}+\tau_{i j, s}\right) .
$$

В случае нормального падения на такую среду одну из составляющих поля можно исключить с помощью уравнения $\operatorname{div} \mathbf{D}=0$ и представить двумерное волновое уравнение в следующем виде [13-15]:

$$
\frac{d^{2} \mathbf{E}(z)}{d z^{2}}+\frac{\omega^{2}}{c^{2}} \hat{\tilde{\varepsilon}}(z) \mathbf{E}(z)=0, \quad i, j=x, y .
$$

Здесь $\mathbf{E}=\left(E_{x} E_{y}\right)^{T}$ - двумерный вектор Джонса, $\hat{\tilde{\varepsilon}}(z)-$ комплексная диэлектрическая проницаемость (2) такой среды. Допустим, что среда является слабо неоднородной, т. е. изменения $\varepsilon_{i j}(z)$ и $\tau_{i j}(z)$ на длине волны малы. В рассматриваемой задаче это условие выполняется практически всегда. Кратко обсудим вывод укороченных уравнений в такой среде (подробности можно найти в [14]). Чтобы разделить быстрые и медленные изменения поля, решение (3) следует искать в следующем виде:

$$
\mathbf{E}(z)=\mathbf{E}_{0}(z) \exp \{i \phi(z)\}
$$

В (4) $\mathbf{E}_{0}(z)$ - медленно меняющаяся комплексная амплитуда, а фаза

$$
\phi(z)=\frac{\omega}{c} \int \sqrt{\frac{\varepsilon_{x x, s}+\varepsilon_{y y, s}}{2}} d z .
$$

Подставив (4) в основное уравнение (3) и учитывая медленность изменения $\mathbf{E}_{0}(z)$, в геометрооптическом приближении (т.е. пренебрегая слагаемым $\left.d^{2} \mathbf{E}_{0}(z) / d z^{2}\right)$, получим следующее векторное уравнение:

$$
\frac{d \mathbf{J}(z)}{d z}=\frac{i}{2} \hat{H}(z) \mathbf{J}(z),
$$

где для сокращения записи введены обозначения

$$
\hat{H}(z)=\frac{1}{\phi^{\prime}}\left\{\frac{\omega^{2}}{c^{2}} \tilde{\tilde{\varepsilon}}(z)-\left(\phi^{\prime}\right)^{2} \hat{I}\right\}, \quad \mathbf{J}(z)=\sqrt{\phi^{\prime}} \mathbf{E}_{0}(z) .
$$

Здесь

$$
\hat{I}=\left(\begin{array}{ll}
1 & 0 \\
0 & 1
\end{array}\right)
$$

- единичная матрица, а штрих означает дифференцирование по продольной координате $z$. Подставляя выражение (2) в (5b), получим

$$
\hat{H}(z)=\left(\begin{array}{cc}
\Omega_{1}+i\left(T_{0}+T_{1}\right) & \Omega_{2}+i \Omega_{3}+i\left(T_{2}+i T_{3}\right) \\
\Omega_{2}-i \Omega_{3}+i\left(T_{2}-i T_{3}\right) & -\Omega_{1}+i\left(T_{0}-T_{1}\right)
\end{array}\right) .
$$


Здесь

$$
\begin{gathered}
\Omega_{1}=\frac{\omega^{2}}{c^{2}} \frac{\varepsilon_{x x, s}-\varepsilon_{y y, s}}{2 \phi^{\prime}}, \quad \Omega_{2}=\frac{\omega^{2}}{c^{2}} \frac{\varepsilon_{x y, s}}{\phi^{\prime}}, \\
\Omega_{3}=\frac{\omega^{2}}{c^{2}} \frac{\varepsilon_{x y, a}}{\phi^{\prime}} . \\
T_{0}=\frac{\omega^{2}}{c^{2}} \frac{\sigma_{x x, s}+\sigma_{y y, s}}{2 \phi^{\prime}}, \quad T_{1}=\frac{\omega^{2}}{c^{2}} \frac{\sigma_{x x, s}-\sigma_{y y, s}}{2 \phi^{\prime}}, \\
T_{2}=\frac{\omega^{2}}{c^{2}} \frac{\sigma_{x y, s}}{\phi^{\prime}}, \quad T_{3}=\frac{\omega^{2}}{c^{2}} \frac{\sigma_{x y, a}}{\phi^{\prime}} .
\end{gathered}
$$

Это наиболее общая система связанных уравнений, которая позволяет анализировать распространение световой волны в неоднородно анизотропных и поглощающих средах в приближении геометрической оптики.

\section{Методика решения}

Запишем систему уравнений (5) в явном виде

$$
\left\{\begin{array}{l}
\frac{d J_{x}(z)}{d z}=\frac{i}{2}\left\{\left(\Omega_{1}+i T_{1}\right) J_{x}(z)+T_{0} J_{x}(z)+\left(\Omega_{2}+i T_{2}\right) J_{y}(z)\right\}, \\
\frac{d J_{y}(z)}{d z}=\frac{i}{2}\left\{\left(\Omega_{2}+i T_{2}\right) J_{x}(z)+T_{0} J_{y}(z)-\left(\Omega_{1}+i T_{1}\right) J_{y}(z)\right\} .
\end{array}\right.
$$

Путем элементарной подстановки

$$
J_{x, y}(z)=f_{x, y}(z) \exp \left(i \frac{T_{0}}{2} z\right)
$$

можно исключить слагаемые, содержащие $T_{0}$. В результате имеем

$$
\left\{\begin{array}{l}
\frac{d f_{x}(z)}{d z}=\frac{i}{2}\left\{\left(\Omega_{1}+i T_{1}\right) f_{x}(z)++\left(\Omega_{2}+i T_{2}\right) f_{y}(z)\right\}, \\
\frac{d f_{y}(z)}{d z}=\frac{i}{2}\left\{\left(\Omega_{2}+i T_{2}\right) f_{x}(z)-\left(\Omega_{1}+i T_{1}\right) f_{y}(z)\right\}
\end{array}\right.
$$

Применим полученную систему уравнений для анализа распространения линейно поляризованного света в средах с различными оптическими характеристиками. Как известно $[4,19]$, если в НЖК („хозяин“) растворен плеохроичный краситель („гость“), молекулы которого обладают анизотропией поглощения, то при ориентации НЖК молекулы красителя тоже будут ориентированы. С учетом сказанного тензор диэлектрической проницаемости комбинированной среды можно представить в следующем виде:

$$
\begin{gathered}
\hat{\varepsilon}(z)=\left(\begin{array}{cc}
\varepsilon_{\perp}+\varepsilon_{a} \cos ^{2} \psi(z) & \varepsilon_{a} \sin \psi(z) \cos \psi(z) \\
\varepsilon_{a} \sin \psi(z) \cos \psi(z) & \varepsilon_{\perp}+\varepsilon_{a} \sin ^{2} \psi(z)
\end{array}\right), \\
\hat{\tau}(z)=\left(\begin{array}{cc}
\tau_{\perp}+\tau_{a} \cos ^{2} \psi(z) & \tau_{a} \sin \psi(z) \cos \psi(z) \\
\tau_{a} \sin \psi(z) \cos \psi(z) & \tau_{\perp}+\tau_{a} \sin ^{2} \psi(z)
\end{array}\right) .
\end{gathered}
$$

Здесь $\psi(z)-$ угол между локальной ориентацией директора и осью $x$ лабораторной системы координат (рис. 1), $\varepsilon_{a}=\varepsilon_{\|}-\varepsilon_{\perp}, \tau_{a}=\tau_{\|}-\tau_{\perp}$, а $\varepsilon_{\|}, \varepsilon_{\perp}, \tau_{\|}, \tau_{\perp}$ - главные значения тензора диэлектрической проницаемости и поглощения. Тогда, согласно определению (6), имеем

$$
\begin{gathered}
\frac{d \mathbf{f}(z)}{d z}=i \gamma \hat{H}(z) \mathbf{f}(z), \\
\hat{H}(z)=\left(\begin{array}{cc}
\cos 2 \psi(z) & \sin 2 \psi(z) \\
\sin 2 \psi(z) & -\cos 2 \psi(z)
\end{array}\right), \\
\gamma=\frac{\pi\left(\varepsilon_{a}+i \tau_{a}\right)}{2 \lambda n_{0}}, \quad n_{0}=\sqrt{\frac{\varepsilon_{\|}+\varepsilon_{\perp}}{2}} .
\end{gathered}
$$

Система уравнений (10) позволяет полностью исследовать эволюцию состояния поляризации света в рассматриваемой среде. Непосредственное решение системы (10) с переменными коэффициентами связано с определенными трудностями. Однако в случае линейного закона изменения $\psi(z)$ решение существенно упрощается, и удается получить аналитическое решение задачи, если перейти во вращающуюся систему координат посредством преобразования $[13,14]$

$$
\mathbf{f}(z)=\hat{R}^{-1} \mathbf{A}(z), \quad \hat{H}(z)=\hat{R}^{-1} \hat{H}_{0} \hat{R},
$$

где $\mathbf{A}=\left(A_{\xi} A_{\eta}\right)^{T}-$ вектор Джонса во вращающейся системе координат, $\hat{R}(\psi)$ - матрица поворота координатных осей:

$$
\hat{R}(\psi)=\left(\begin{array}{cc}
\cos \psi(z) & \sin \psi(z) \\
-\sin \psi(z) & \cos \psi(z)
\end{array}\right)
$$

матрица

$$
\hat{H}_{0}=\left(\begin{array}{cc}
1 & 0 \\
0 & -1
\end{array}\right)
$$

- характерная матрица данной среды в локальной системе координат, $\hat{R}^{-1}(\psi)$ - обратная матрица.

После несложных, но громоздких преобразований получим следующую систему связанных уравнений относительно амплитуд $A_{\xi, \eta}$ :

$$
\left\{\begin{array}{l}
\frac{d A_{\xi}(z)}{d z}=i \gamma A_{\xi}(z)+\alpha A_{\eta}(z), \\
\frac{d A_{\eta}(z)}{d z}=-\alpha A_{\xi}(z)-i \gamma A_{\eta}(z) .
\end{array}\right.
$$

Система (12) представляет систему уравнений с постоянными коэффициентами, решение которой хорошо известно [20]. С учетом этого решения и после обратного преобразования в лабораторную систему координат полное аналитическое решение задачи представляется в следующем виде:

$$
\left.\begin{array}{l}
E_{x}(z)=\frac{i}{2 \Omega}\left[C_{x}(z) e^{-i \Omega z}+D_{x}(z) e^{i \Omega z}\right] \exp ^{\left(i \frac{2 \pi}{\lambda} n_{0}-\frac{T_{0}}{2}\right) z}, \\
E_{y}(z)=\frac{i}{2 \Omega}\left[C_{y}(z) e^{-i \Omega z}+D_{y}(z) e^{i \Omega z}\right] \exp ^{\left(i \frac{2 \pi}{\lambda} n_{0}-\frac{T_{0}}{2}\right)} z
\end{array}\right\}
$$

Введенные для сокращения записи обозначения имеют следующий вид:

$$
\begin{aligned}
& C_{x}(z)=\left[\left(\cos \psi_{0}-i \frac{\alpha}{\gamma+\Omega} \sin \psi_{0}\right) B_{0}\right. \\
& \left.-\left(\sin \psi_{0}+i \frac{\alpha}{\gamma+\Omega} \cos \psi_{0}\right) A_{0}\right][\alpha \cos \psi(z)+i(\gamma+\Omega) \sin \psi(z)],
\end{aligned}
$$



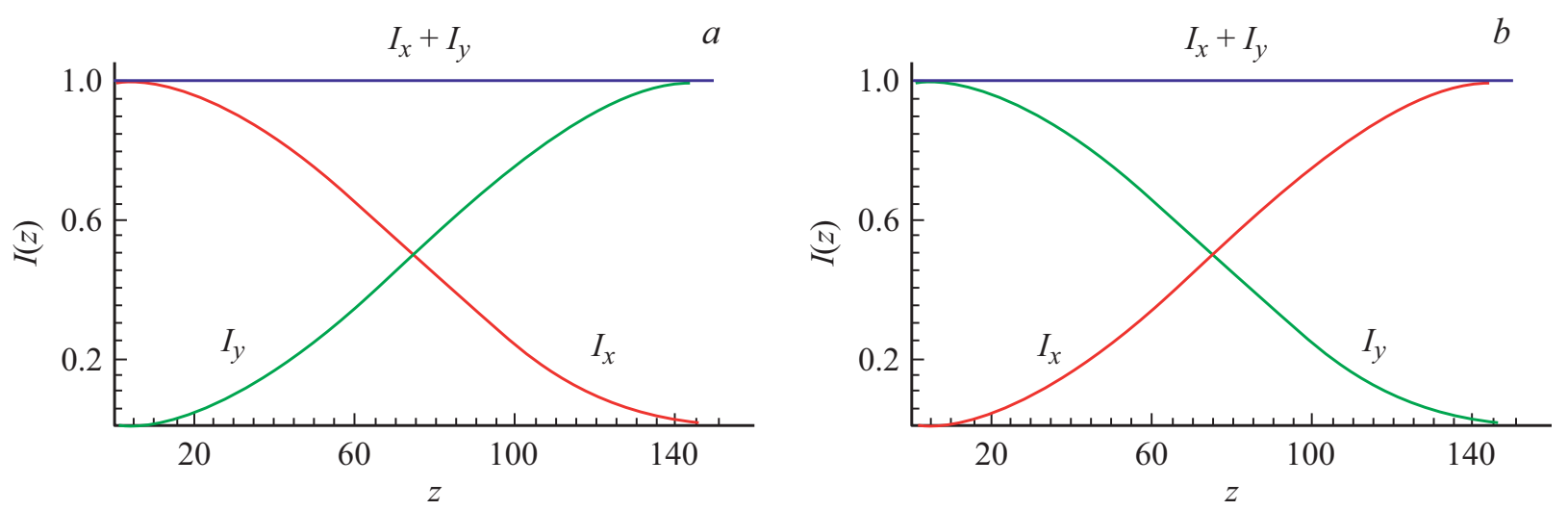

Рис. 2. Эволюция декартовых составляющих поляризации света в твист-нематике МББА (поглощение отсутствует). Значения параметров следующие: $\varepsilon_{P}=3.24, \varepsilon_{\perp}=2.434, \lambda=0.53 \mu \mathrm{m}$, толщина слоя ЖК составляет $150 \mu$ m. Азимут $\beta=0(a), \pi / 2(b)$.
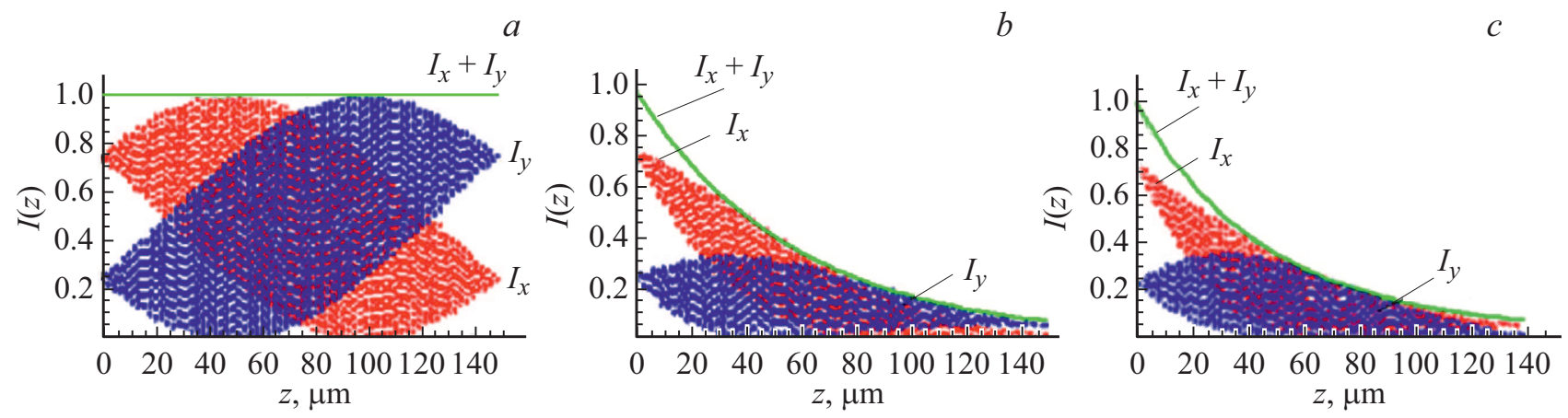

Рис. 3. Эволюция декартовых составляющих поляризации света в твист-нематике МББА (поглощение отсутствует). Значения параметров следующие: $\varepsilon_{P}=3.24, \varepsilon_{\perp}=2.434, \lambda=0.53 \mu \mathrm{m}$, толщина слоя ЖК составляет $150 \mu$ m, азимут $\beta=\pi / 6:(a)$ поглощение отсутствует, $(b)$ в присутствии изотропного поглощения, $\tau_{\|}=\tau_{\perp}=2.5 \cdot 10^{-3},(c)$ в присутствии анизотропного поглощения, $\tau_{\|}=3.5 \cdot 10^{-3}, \tau_{\perp}=1.5 \cdot 10^{-3}$.

$D_{x}(z)=\left[\left(\alpha \sin \psi_{0}-i(\gamma+\Omega) \cos \psi_{0}\right) A_{0}\right.$

$\left.-\left(\alpha \cos \psi_{0}+i(\gamma+\Omega) \sin \psi_{0}\right) B_{0}\right]\left[\cos \psi(z)-i \frac{\alpha}{\gamma+\Omega} \sin \psi(z)\right]$,

$C_{y}(z)=\left[\left(\cos \psi_{0}-i \frac{\alpha}{\gamma+\Omega} \sin \psi_{0}\right) B_{0}\right.$

$\left.-\left(\sin \psi_{0}+i \frac{\alpha}{\gamma+\Omega} \cos \psi_{0}\right) A_{0}\right][\alpha \sin \psi(z)-i(\gamma+\Omega) \cos \psi(z)]$,

$D_{y}(z)=\left[\left(\alpha \sin \psi_{0}-i(\gamma+\Omega) \cos \psi_{0}\right) A_{0}\right.$

$\left.-\left(\alpha \cos \psi_{0}+i(\gamma+\Omega) \cos \psi_{0}\right) A_{0}\right]\left[\sin \psi(z)+i \frac{\alpha}{\gamma+\Omega} \cos \psi(z)\right]$.

Здесь $\Omega^{2}=\gamma^{2}+\alpha^{2}$, а граничные условия имеют следующий вид:

$$
A_{0}=E_{x}(z=0), \quad B_{0}=E_{y}(z=0)
$$

Величины $A_{0}$ и $B_{0}$ могут быть и комплексными (для возможности анализа эллиптически поляризованной входной волны). В случае линейной поляризации $A_{0}=\cos \beta$, $B_{0}=\sin \beta$, где $\beta-$ азимут поляризации, т. е. угол, который составляет поляризация с плоскостью $x z$. Нетрудно проверить, что $(13)$ в пределе $\alpha \rightarrow 0, \tau \rightarrow 0$ переходят в хорошо известные выражения [1].

\section{Обсуждение результатов}

С помощью системы (13) проанализируем прохождение линейно поляризованной плоской волны через слой твист-ориентированного НЖК с примесями молекул красителя.

В качестве примера рассмотрим НЖК МББА, в который добавлены молекулы красителя 2-(3-этилбензотиазолиден)-2-бутенилиден-3-окситианафтена. Параметры МББА и красителя хорошо известны $[4,19]$. Ограничимся случаем, когда вращение локальной ориентации директора линейно, т. е. азимутальный угол как для ЖК, так и для молекул красителя меняется согласно закону $\psi(z)=\alpha z$. Рассмотрим распространение линейно поляризованного излучения сквозь такую комбинированную среду.

Как известно, в такой среде в отсутствие поглощения наблюдается явление адиабатического отслеживания $[6,14]$, т.е. если падающая волна на входе в среду линейно поляризована вдоль одной из нормальных волн, 

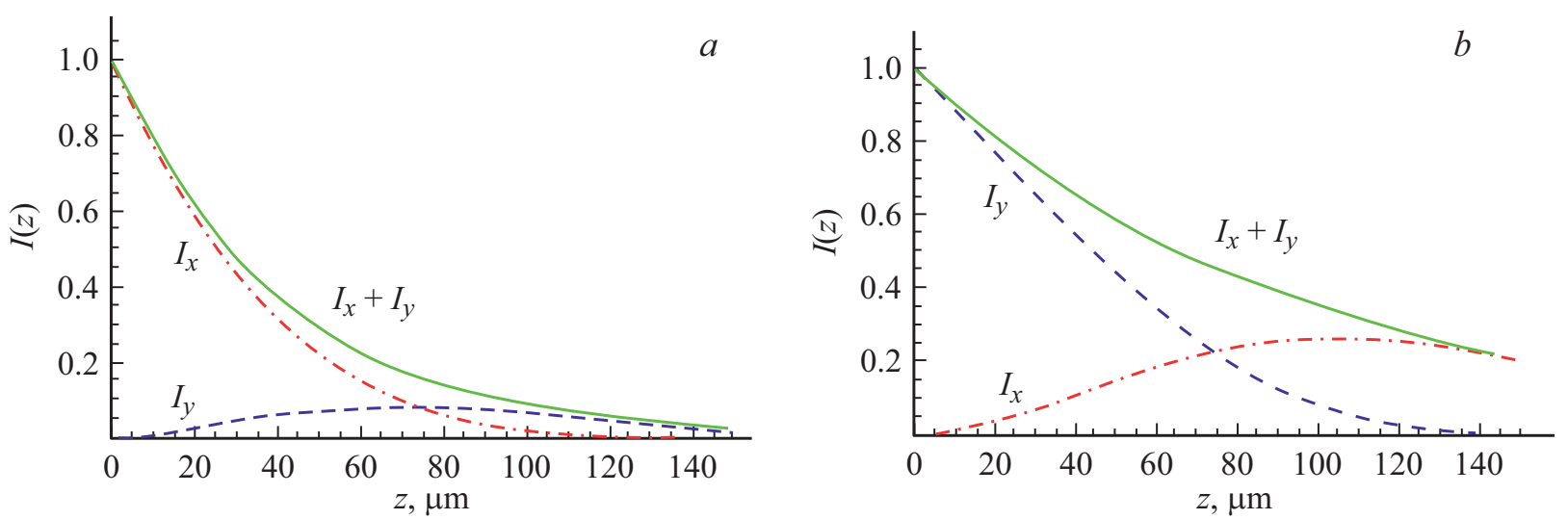

Рис. 4. Эволюция декартовых составляющих поляризации света в твист-нематике МББА (в присутствии анизотропного поглощения). Значения параметров следующие: $\varepsilon_{P}=3.24, \varepsilon_{\perp}=2.434, \lambda=0.53 \mu \mathrm{m}$, толщина слоя ЖК составляет $150 \mu \mathrm{m}$, $\tau_{\|}=3.5 \cdot 10^{-3}, \tau_{\perp}=1.5 \cdot 10^{-3} \cdot \beta=0(a), \pi / 2(b)$.

то вектор поляризации световой волны отслеживает вращение главных осей при условии, что коэффициент кручения мал. Сказанное демонстрируется на рис. 2. Если азимутальный угол поляризации отличается от 0 и $\pi / / 2$, картина содержит множество осцилляций, но тем не менее явление отслеживания сохраняется (рис. 3,a). При добавлении молекул красителя, однако, проявляются некоторые особенности. Особо следует подчеркнуть существнную роль анизотропии поглощения. В качестве примера на рис. $3, b, c$ представлены зависимости $x$ - и $y$-составляющих интенсивностей от продольной координаты. Сравнение этих рисунков демонстрирует роль анизотропии поглощения. Если в случае изотропного поглощения (рис. $3, b$ ) сохраняются такие же зависимости, как и в случае отсутствия поглощения (рис. 3,a), а именно $I_{y}>I_{x}$, если $I_{0 x}>I_{0 y}$, то в случае анизотропного поглощения картина обратная (рис. 2, $b$ ). Следует отметить, что в случае азимутального угла $\beta>\pi / 2$ такая аномалия отсутствует. Обратим внимание еще на одну особенность анизотропного поглощения. На рис. 4 представлены зависимости $x$ - и $y$-составляющих интенсивностей от продольной координаты в двух предельных случаях $\beta=0$ и $\beta=\pi / 2$. Хотя толщины образцов и коэффициенты анизотропного поглощения в обоих случаях одинаковые, тем не менее в случае $\beta=\pi / 2$ суммарное ослабление меньше.

\section{Заключительные замечания}

Таким образом, полученные в настоящей работе выражения позволяют количественно исследовать эволюцию состояния поляризации света в твист-ориентированном нематике с анизотропно поглощающим красителем. Сложная картина распространения и особенности поведения связаны с процессами конкуренции отслеживания и изменения коэффициента поглощения вдоль продольной координаты.
Можно сделать и другой вывод. При решении системы связанных уравнений переход во вращающуюся систему координат позволяет получить полное аналитическое решение задачи, что может быть полезным и при исследовании других (и не только оптических) задач. Такой подход может быть особенно плодотворным также в средах с зависящей от времени анизотропией с целью создания поляризационных устройств с временным управлением [21].

\section{Конфликт интересов}

Авторы заявляют, что у них нет конфликта интересов.

\section{Список литературы}

[1] Yariv A., Yeh P. Optical Waves in Crystals. Wiley, 1984.

[2] Иванов О.В. Распространение электромагнитных волн в анизотропных и бианизотропных слоистых структурах. Ульяновск: УлГТУ, 2010

[3] Федоров Ф.И. Оптика анизотропных сред. М.: Едиториал, УРCC, 2004

[4] Blinov L.M. Structure and Properties of Liquid Crystals. Springer, 2011.

[5] Collett E., Schaefer B. Polarized Light for Scientists and Engineers. Polarwave Group, 2012. 802 p.

[6] Yeh P., Gu C. Optics of Liquid Crystal Displays. Wiley, 1999. $438 \mathrm{p}$.

[7] Scharf T. Polarized Light in Liquid Crystals and Polymers. Wiley, 2006. $400 \mathrm{p}$.

[8] Allen L., Eberli J.H. Optical Resonance and Two-Level Atoms. NY: John Wiley and Sons, 1975.

[9] Segre S.E. // J. Phys. D. 2003. V. 36. P. 2806-2810.

[10] Segre S.E. // JOSA. A. 2000. V. 17. P. 95.

[11] Kubo H., Nagata R. // JOSA. 1985. V. 73. P. 1719-1724.

[12] Kravtsov Yu.A., Bieg B. // Centr. Eur. J. Phys. 2008. V. 6. P. 563.

[13] Aslanyan A.L., Aslanyan L.S., Chilingaryan Yu.S. // Opt. Spectrosc. 2014. V. 116. N 3. P. 483-487. 
[14] Aslanyan A.L., Aslanyan L.S., Alaverdyan R.B., Gevorgyan G.S., Nersisyan S.Ts. // J. Contemp. Phys. (Armenian Acad. Sci.). 2015. V. 50. N 2. P. 137-143.

[15] Aslanyan L.S. // J. Contemp. Phys. (Armenian Acad. Sci.). 2017. V. 52. N 3. P. 279-288.

[16] Yanossy I. // Phys. Rev. E. 1994. V. 49. P. 2957-2963.

[17] Muenster R., Jarash M., Zhuang X., Shen Y.R. // Phys. Rev. Lett. 1997. V. 78. P. 42-45.

[18] Агранович В.М., Гинзбург В.Л. Кристаллооптика с учетом пространственной дисперсии и теория экситонов. М.: Наука, 1979.

[19] Сонин А.С. Введение в физику жидких кристаллов. М.: Наука, 1983.

[20] Андронов А.А., Витm А.А., Хайкин С.Э. Теория колебаний. М.: Наука, 1981. 568 c.

[21] Tudor T. // J. Phys. Soc. Japan. 2012. V. 81. P. 024006. 